\title{
MacroH2A suppresses the proliferation of the B16 melanoma cell line
}

\author{
SHAORONG LEI, JIANHONG LONG and JIAGUANG LI
}

\author{
Department of Plastic Surgery, Xiangya Hospital, Central South University, Changsha, Hunan 410008, P.R. China
}

Received July 5, 2013; Accepted March 27, 2014

DOI: $10.3892 / \mathrm{mmr} .2014 .2482$

\begin{abstract}
MacroH2A is the most frequently altered histone, which participates in cancer progression. Increasing evidence demonstrates that cancer progression could be regulated by macroH2A by affecting the cell cycle. In the present study, it was demonstrated that macroH2A suppresses melanoma cell progression and the molecular mechanisms underlying this process were examined. The interference and overexpression vectors of macroH $2 \mathrm{~A}$ were constructed and then transferred into B16 melanoma cells and, following transfection, were analyzed by quantitative polymerase chain reaction (PCR), western blot analysis and immunofluorescence assays. Apoptosis and the cell cycle stage among all the treatment groups were detected. Then, cyclin D1, cyclin D3, cyclin-dependent protein kinase (CDK) 4, CDK6 and CDK8 expression was detected in order to elucidate the effects of macroH2A on cell cycle-related genes. The results demonstrated that the overexpression of macroH2A suppressed melanoma cell progression and arrested the cells in the G2/M stage. Furthermore, macroH2A inhibits cyclin D1, cyclin D2, CDK6 and CDK8 expression in B16 melanoma cells. In conclusion, the results demonstrated that macroH2A, a critical component of chromatin, suppresses the development of melanoma (which results from a disordered cell cycle) through regulating cyclin D1, cyclin D3 and CDK6 genes.
\end{abstract}

\section{Introduction}

At present, cancer is regarded as a disease that is caused by genetic and epigenetic alterations (1). Increasing evidence demonstrates that chromatin-mediated changes induce cancer, including DNA methylation, histone variants and miRNA variation (2). Once histone variants are replaced by normal

Correspondence to: Professor Jianhong Long, Department of Plastic Surgery, Xiangya Hospital, Central South University, 1 Xiangya Road, Changsha, Hunan 410008, P.R. China

E-mail: jianhonglong1962@126.com

Key words: macroH2A, melanoma, cell cycle, cell proliferation, cyclin D histones, the chromatin is changed. Subsequently, unfolded and remodeled chromatin controls access to the transcription start site, which regulates gene transcription (3). Additionally, the correlation between these changes and cancer progression has been established (4). Histone variants affect stable gene expression, which participates in tumor progression and differentiation (5).

Histones, which are highly conserved in mammals, are crucial in regulating nuclear activation via changing the chromatin structure $(6,7)$. Yet, the mechanisms by which they change chromatin structure are less understood. $\mathrm{H} 3$ and $\mathrm{H} 2 \mathrm{~A}$, two major variants associated with several tissue-restricted proteins, have been revealed to be involved in this process (8). In these variants, due to its canonical counterpart, macroH2A demonstrates a different characterization. MacroH2A, the only histone with a tripartite structure consisting of an N-terminal histone-fold, contains an unstructured linker domain and a unique C-terminal macro domain $(9,10)$. Due to its structure, macroH2A demonstrates the most frequent alterations among all the histone variants $(11,12)$. Kapoor et al $(13)$ demonstrated that the loss of macroH2A is associated with chromatin condensation and regulated gene expression during melanoma developmental programs. Furthermore, by knocking down macroH2A in melanoma cells, it was confirmed that the loss of macroH2A promoted cancer development via the transcriptional upregulation of CDK8. CDK8 is a member of the cyclin-dependent protein kinase (CDK) family, which has regulatory functions in the cell cycle $(14,15)$. This finding suggests that macroH2A affects melanoma progression through changes in the cell cycle. However, to date, information is lacking regarding the effects of macroH2A on cell cycle regulatory genes.

The cell cycle involves strict events that control cell division and proliferation. The key factors, including CDKs and cyclins, trigger the transition in the process of the cell cycle (16-18). During this event, dysfunctional expression of genes induced by normal metabolic activity or environmental factors arrested or delayed checkpoints prior to cell division $(19,20)$. In addition, $\mathrm{CDK} /$ cyclin B is important in stabilizing the genome in $\mathrm{S}$ phase $(21,22)$. Thus, abnormal expression of these factors disorganized the process of cell division $(23,24)$.

In the present study, the effects of macroH2A on cell cycle-related genes were examined in melanoma. MacroH2A interference, overexpression, overexpression rescue and interference rescue treatments were performed by the transfection 
of short hairpin (sh)RNA vectors and/or overexpression vectors. Following transfection, macroH2A expression was determined. By employing flow cytometry, the regulation of the B16 melanoma cell line proliferation by macroH2A was confirmed. Furthermore, the regulation of macroH2A on cyclin D and CDK were analyzed in the B16 melanoma cell line. The present study provides insights into the effects of macroH2A on melanoma progression.

\section{Materials and methods}

Ethics statement. The present study obtained ethics approval from the ethics committee at Xiangya Hospital, Central South University (Changsha, China). The storage of samples for exploratory immunological analyses was also ethically approved.

Cell culture. The B16 mouse melanoma cells were obtained from the American Type Culture Collection (Manassas, VA, USA). The cells were grown in RPMI-1640 medium supplemented with $10 \%$ fetal bovine serum (Invitrogen Life Technologies, Carlsbad, CA, USA) in $5 \% \mathrm{CO}_{2}$ and saturated humidity at $37^{\circ} \mathrm{C}$. Cell viability was estimated by trypan blue exclusion. For cell morphology examination, cells were grown on a chambered coverglass system (Thermo Scientific, Rockford, IL, USA) and observed with an inverted microscope (Nikon Corporation, Tokyo, Japan).

Vector design and transfection. For the comparison of biophysical properties following knockdown and overexpression of macroH2 $\mathrm{A}$, the macroH $2 \mathrm{~A}$ interference and overexpression vectors were constructed. The recombinant expression plasmid (macroH2A shRNA plasmid) was purchased from Santa Cruz Biotechnology, Inc. (Santa Cruz, CA, USA; sc-62576-SH). The recombinant expression plasmid expressing macroH2A was constructed. Briefly, the open reading frame of macroH2A (GenBank accession no. NM012015) was cloned into the pcDNA3.1(t) plasmid (Invitrogen Life Technologies) between XhoI and BamHI sites to build recombinant $\mathrm{pc} 3.1 \mathrm{~h}$ macroH2A plasmids. The cells were transfected with pcDNA3.1(t)-macroH2A and/or the macroH2A shRNA plasmid using Lipofectamine ${ }^{\mathrm{TM}} 2000$ (Invitrogen Life Technologies) according to the manufacturer's instructions. Following $24 \mathrm{~h}$ of transfection, the cells were harvested and used for the following experiments.

The cells were randomly divided into five groups (five parallel treatments per group), including the control group (non-treated group), macroH2A interference group (1 $\mu \mathrm{g}$ macroH2A shRNA plasmid transfection), macroH2A overexpression group (1 $\mu \mathrm{g}$ pcDNA3.1(t)-macroH2A plasmid transfection), macroH2A overexpression rescue group $(1 \mu \mathrm{g}$ pcDNA3.1(t)-macroH2A plasmid transfection for $12 \mathrm{~h}$ following $1 \mu \mathrm{g}$ macroH2A shRNA plasmid transfection for $12 \mathrm{~h})$ and the macroH2A interference rescue group (1 $\mu \mathrm{g}$ macroH2A shRNA plasmid transfection for $12 \mathrm{~h}$ following $1 \mu \mathrm{g}$ pcDNA3.1(t)-macroH2A plasmid transfection for $12 \mathrm{~h}$ ).

Quantitative polymerase chain reaction ( $q P C R)$. In order to analyze mRNA expression among different groups, qPCR was performed. All the primers and probes were designed by
Applied Biosystems (Foster City, CA, USA), which hybridized between exons to avoid genomic DNA amplification. Total RNA isolation was performed using RNA TRIzol according to the manufacturer's instructions (Invitrogen Life Technologies). By using the cDNA library construction kit (Clontech, Mountain View, CA, USA), $1 \mu \mathrm{g}$ of total RNA was used to synthesize cDNA according to the manufacturer's instructions (Takara Bio, Inc., Shiga, Japan). The transcriptional levels of macroH2A, cyclin D1, cyclin D3, CDK4, CDK6, CDK8 and GAPDH (a housekeeping gene) were quantified using the ABI 7500 real-time PCR system (Applied Biosystems). The amplification conditions were as follows: $95^{\circ} \mathrm{C}$ for $10 \mathrm{~min}$, followed by 40 cycles of $15 \mathrm{sec}$ at $95^{\circ} \mathrm{C}$ and $1 \mathrm{~min}$ at $60^{\circ} \mathrm{C}$, using the TaqMan ${ }^{\circledR}$ Universal PCR Master mix (Applied Biosystems). All the results were normalized to the levels of GAPDH RNA (TaqMan probes; Applied Biosystems). The relative expression level was calculated using the $2^{-\Delta \Delta \mathrm{Ct}}$ method.

Western blot analysis. Samples were separated in $10 \%$ SDS-PAGE gels and transferred to polyvinylidene fluoride membranes (Millipore, Billerica, MA, USA). Following inhibition with 4\% non-fat milk, macroH2A, cyclin D1, cyclin D3, CDK4, CDK6 and CDK8 were detected by incubation with the monoclonal anti-macroH2A antibody produced from rabbit (ab83782; Abcam, Cambridge, MA, USA), anti-cyclin D1 antibody produced from rabbit (ab7958; Abcam), anti-cyclin D3 antibody produced from rabbit (ab112034; Abcam), anti-CDK4 antibody produced from rabbit (ab7955; Abcam), anti-CDK6 antibody produced from rabbit (ab151247, Abcam) and anti-CDK8 antibody produced from rabbit (ab123940; Abcam). GAPDH (a housekeeping gene) was detected by the monoclonal anti-GAPDH antibody (ab9485, Abcam). The anti-mouse IgG secondary antibody conjugated to horseradish peroxidase (Amersham Biosciences, Uppsala, Sweden) and an enhanced chemiluminescent substrate (ECL plus; Amersham Pharmacia Biotech, Piscataway, NJ, USA) were used for signal development. Images were captured using a Fujifilm FLA-5000 image reader (Fujifilm, Stanford, CT, USA).

Immunofluorescence. B16 melanoma cells were grown on $24 \times 24-\mathrm{mm}$ cover glasses and then fixed in $4 \%$ paraformaldehyde solution in phosphate-buffered saline (PBS) for $30 \mathrm{~min}$ prior to 30 min incubation with a blocking reagent (5\% fetal bovine serum in PBS). Primary antibodies were incubated with melanoma cells overnight at $4^{\circ} \mathrm{C}$ prior to washing with PBS. Immunofluorescence staining was performed with secondary antibodies conjugated to fluorescein isothiocyanate (F5262; Sigma-Aldrich, St. Louis, MO, USA). A conventional fluorescence microscope (Carl Zeiss, Göttingen, Germany) was used for visualization.

Statistical analysis. All values are presented as the mean \pm standard deviation. Continuous variables that did not have a Gaussian distribution were log transformed. Student's t-test was used to compare differences between groups. One-way analysis of variance was used to determine differences among groups. $\mathrm{P} \leq 0.05$ was considered to indicate a statistically significant difference. If $F$ ratios exceeded the critical value $(\mathrm{P} \leq 0.05)$, the Newman-Keuls post hoc test was performed to compare the groups. 
A

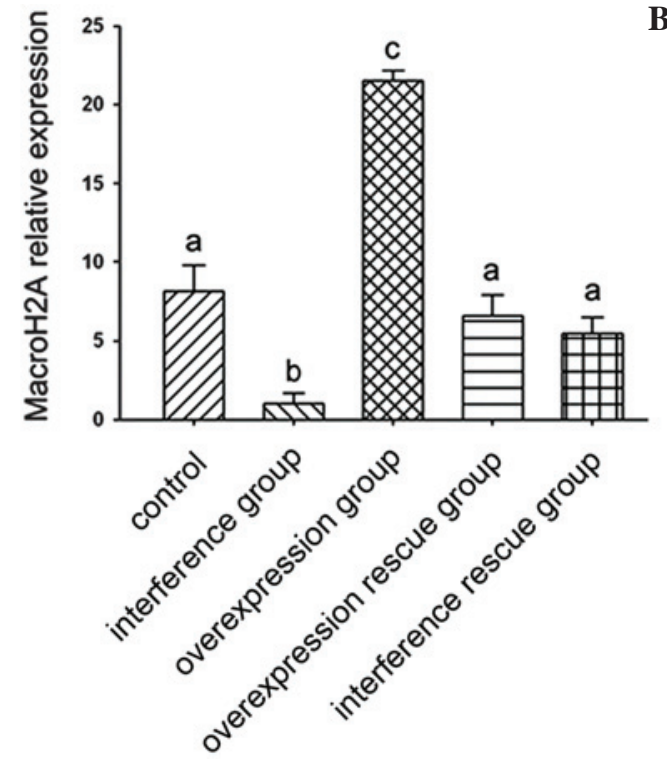

C
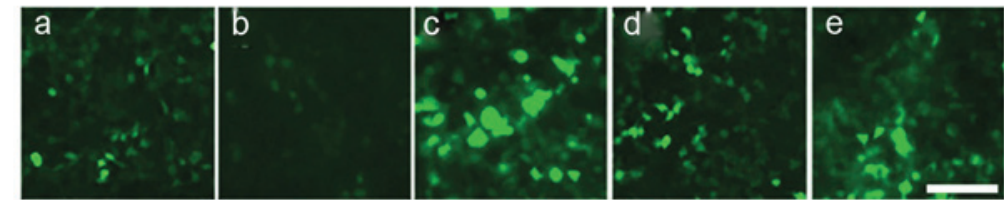

Figure 1. Overexpression and transfer of macroH2A in B16 melanoma cells. (A) macroH2A mRNA expression was analyzed by quantitative polymerase chain reaction (different characters demonstrated significant differences detected by one-way analysis of variance). (B) macroH2A protein expression was analyzed by western blot analysis. (C) macroH2A protein expression was assayed by immunofluorescence. Ca, control; $\mathrm{Cb}$, interference group; Cc, overexpression group; $\mathrm{Cd}$, overexpression rescue group; $\mathrm{Ce}$ interference rescue group.

\section{Results}

Overexpression and transfer of macroH $2 \mathrm{~A}$ in B16 melanoma cells. To detect the effect of macroH2A on B16 melanoma cells, the vectors of macroH2A interference and overexpression were designed and then transferred into B16 melanoma cells. qPCR results demonstrated that the expression levels were the highest in the macroH2A overexpression group followed by the control group, the macroH2A overexpression rescue group and the macroH2A interference rescue group. The lowest expression was identified in the macroH2A interference group (Fig. 1A). Differential protein expression was assayed by western blotting. Similarly, the interference group demonstrated the lowest protein expression while other groups exhibited a higher expression. In addition, the overexpression group demonstrated the highest protein expression among all the groups (Fig. 1B). Immunofluorescence analyses of macroH2A expression in B16 melanoma cells is shown in Fig. 1C. The results also demonstrated the different expression levels of protein among these treatment groups.

Regulation of B16 melanoma cell line proliferation by macroH $2 \mathrm{~A}$. To elucidate the potential cellular regulation of growth inhibition by macroH2A, cell cycle progress and cell apoptosis were examined by flow cytometry (Fig. 2A). Apoptosis was highest in the overexpression group while the apoptosis index was lower in other groups (Fig. 2B). In addition, G2/M arrest rate was analyzed. The overexpression group demonstrated a significantly higher $\mathrm{G} 2 / \mathrm{M}$ arrest rate, which suggested that the overexpression of macroH2A arrested B16 melanoma cells in the G2/M stage (Fig. 2C).

MacroH $2 \mathrm{~A}$ regulates cyclin $\mathrm{D}$ and $\mathrm{CDKs}$. To detect the mechanism of inhibition by macroH2A in B16 melanoma cells, the gene expression of cyclin D and CDKs was assayed following interference or overexpression of macroH2A. Cyclin D1 and cyclin D3 mRNA and protein expression levels were suppressed by the overexpression of macroH2A, while the interference of macroH2A demonstrated no significant difference in the expression of cyclin D1 and promoted the expression of cyclin D3, respectively (Fig. 3A). The overexpression and interference rescue groups demonstrated no difference compared with the control group.

CDKs were also analyzed by qPCR and western blot analysis. No differences among all the treatment groups were identified in the CDK4 expression. However, among all the treatment groups, the overexpression group demonstrated a decrease in CDK6. For CDK8 expression, the interference group demonstrated an increase in expression, while the overexpression group had a decreased expression. Similarly, with cyclin D, the overexpression and interference rescue groups demonstrated no difference compared with the control group (Fig. 3B).

\section{Discussion}

In the present study, the artificial altering of macroH2A expression was performed by the interference and overex- 
A

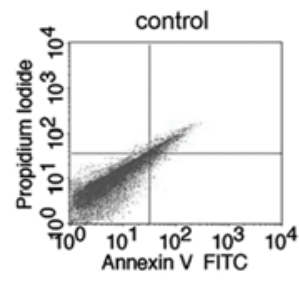

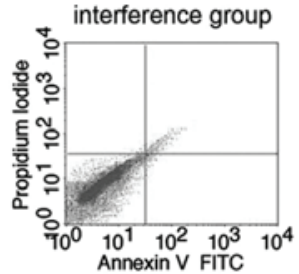

B

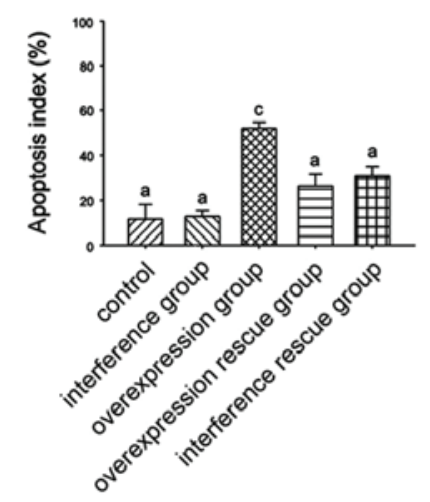

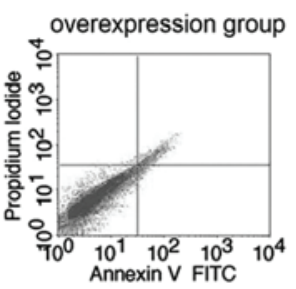

overexpression rescue group interference rescue group
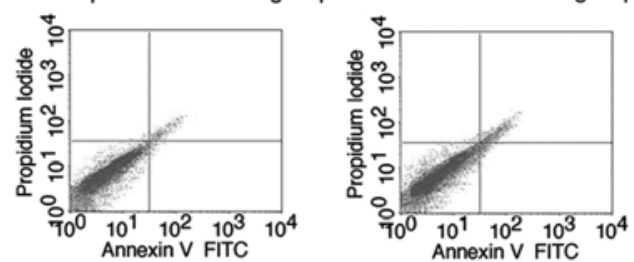

C

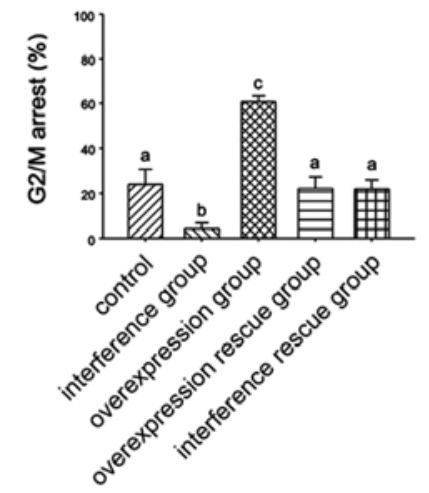

Figure 2. Regulation of melanoma cell line B16 progression by macroH2A. (A) Apoptosis analysis by flow cytometry among all treatment groups. (B) Apoptosis index statistical analysis among all treatment groups. (C) G2/M arrest rates statistical analysis among all the treatment groups. FITC, fluorescein isothiocyanate.

A
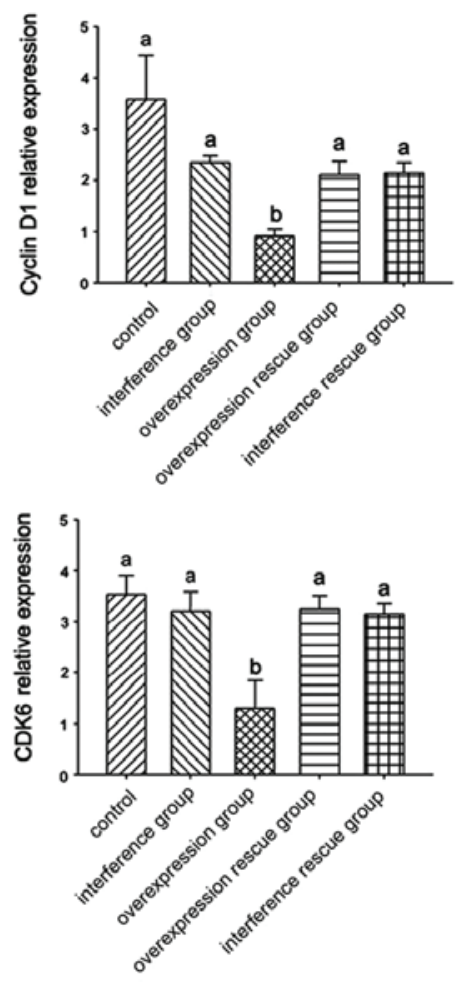
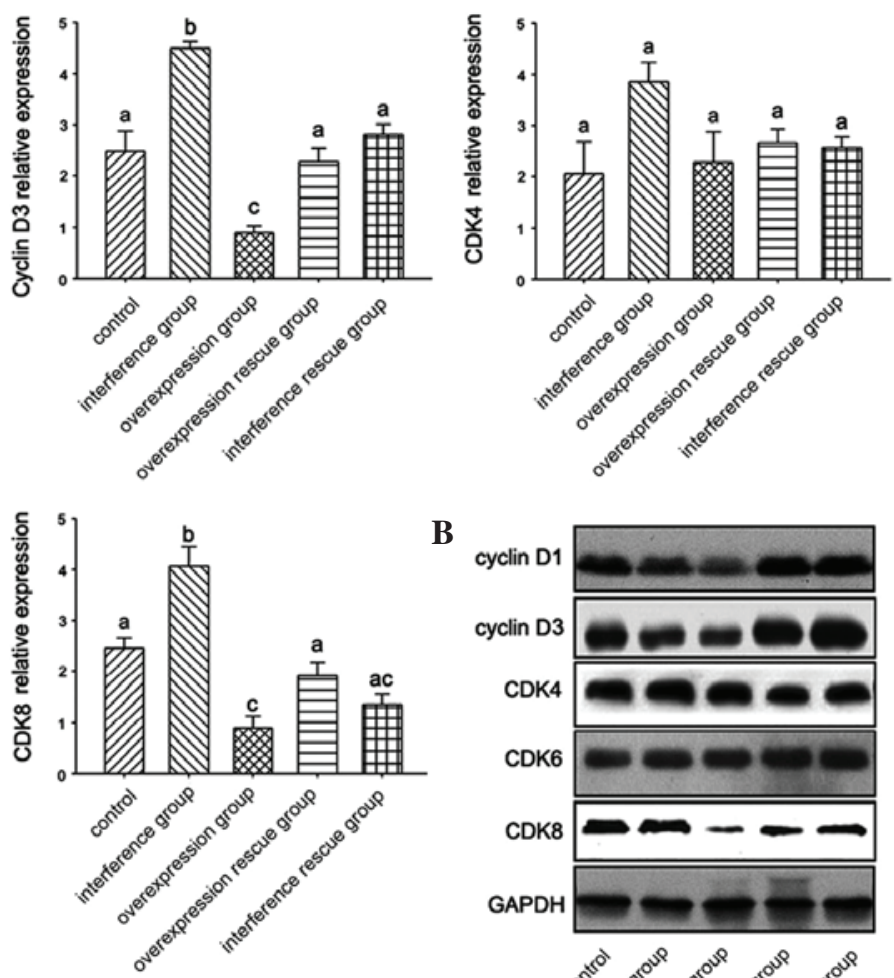

B

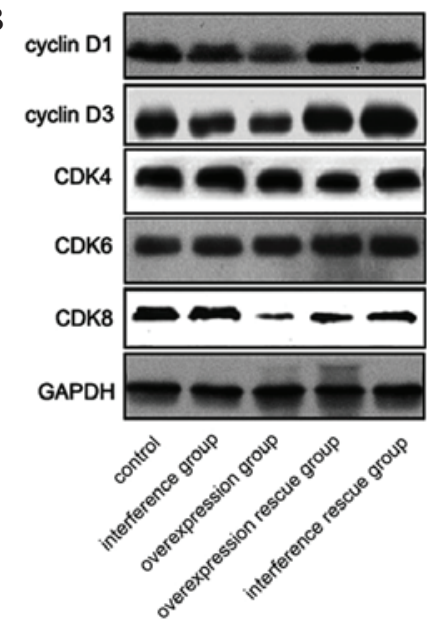

Figure 3. MacroH2A regulates cyclin D and CDK. (A) cyclin D1, cyclin D3, CDK4, CDK6 and CDK8 mRNA expression analyzed by quantitative polymerase chian reaction (different characters demonstrated significant differences detected by one way analysis of variance). (B) cyclin D1, cyclin D3, CDK4, CDK6 and CDK8 protein expression analyzed by western blot analysis. CDK, cyclin-dependent kinase.

pression of macroH2A, as well as overexpression rescue and interference rescue treatments in B16 melanoma cell line progression, in order to evaluate the impact of altered expression on melanoma progression and development. It was demonstrated that macroH2A inhibits the progression of B16 melanoma cells, while macroH2A knockdown promoted 
melanoma progression. Similar results have previously been reported in other forms of human cancer, including testicular, ovarian, lung, bladder, cervical, breast, colon and endometrial cancer (25-30). In these cancer types, the expression levels of macroH2A decreased significantly in tumor tissues compared with normal tissues. In addition, in melanoma, macroH2A expression levels demonstrated a strong negative correlation with tumor development (13). Genome level alterations in macroH2A also affect cancer progression. Dardenne et al (31) elucidated that the alternative splicing of macroH2A induced metastasis in breast cancer cell lines.

The overexpression of macroH2A appears to participate in the development of malignant tumors, based on its well-known inhibitory role in cell cycle progression. In addition, macroH2 A also has a role in genomic stabilization during replication by preventing the occurrence of DNA damage (9). Furthermore, a lack of macroH2A expression led to an increase in CDK8 expression, which may result in cell death through avoiding premature mitotic entry (13). The in vitro results of the present study using overexpression and interference vectors suggested that the upregulation of macroH $2 \mathrm{~A}$ led to increased cell death. It is therefore likely that the low expression levels of macroH2 A observed in melanoma tissues prevent DNA damage and cell death. In accordance with our results, Kapoor et al (13) previously reported that macroH2A suppresses melanoma progression through the regulation of CDK8. In the present study, it was demonstrated that apoptosis of melanoma cells occurs following the overexpression of macroH2A, while no significant differences were identified among other groups. In addition, the G2/M arrest rates were analyzed. The overexpression group demonstrated a significantly higher $\mathrm{G} 2 / \mathrm{M}$ arrest rate, which suggested the overexpression of macroH2A-arrested B16 melanoma cells in the G2/M stage. Thus, the possible explanation of overexpression of macroH2A inducing apoptosis may be that the cell cycle is disorganized by macroH2A. The macroH2A expression changes have been demonstrated through $\mathrm{S}$ phase and G2 toward mitosis. This suggested that in this process, the overexpression of macroH2A arrested melanoma cells in functional G2/M. In melanoma cells, due to a lack of a functional $\mathrm{G} 2 / \mathrm{M}$ checkpoint caused by the depression of macroH2A, the cell cycle progresses into mitosis without securing correct DNA synthesis. Thus, the explanation for the inhibition of the progression of melanoma by macroH2A may be due to its regulatory function in the cell cycle.

In the present study, it was revealed that macroH2A overexpression inhibits cyclin D1, cyclin D3, CDK6 and CDK8 gene expression. In addition, no significant differences were identified in CDK4 among all the treatment groups. It has been reported that cyclin D1 is overexpressed in several cancer types and is regarded to be an oncogene $(32,33)$. Cyclin D1 affects several mechanisms in cancer, including the translocation, amplification and stabilization of mRNA. In addition, elevated cyclin D3 facilitates cancer progression (34). In the present study, it was demonstrated that elevated levels of macroH2A suppressed cyclin D1 and cyclin D3. CDKs, including CDK2, CDK3, CDK4, CDK5, CDK6, CDK7 and CDK8, are crucial in the cell cycle (35). Among them, CDK4, CDK6 and CDK8 are critical for cell proliferation through regulating DNA synthesis at the beginning of the cell cycle and switching the cell cycle from G1 to S phase (36). Since cancer cells often contain high levels of CDK activity, inhibiting CDK gene expression may be a useful therapeutic strategy in cancer treatment. It was confirmed that macroH2A depresses CDK6 and CDK8 gene expression in the B16 cell line. Thus, we hypothesize that other cell cycle-related genes could be regulated by macroH $2 \mathrm{~A}$.

In conclusion, the results indicate that the overexpression of macroH2A induces apoptosis of melanoma cells and arrests the cell in the G2/M stage of the cell cycle. The data further demonstrated that, despite being an inhibitor of cell cycle progression, high expression of macroH2A downregulated cyclin D1, cyclin D3 and CDK6. Thus, the high expression of macroH2A appears to protect the cancer cell from a disordered cell cycle through the regulation of cyclin D1, cyclin D3 and CDK6 genes. The present findings support emerging links between chromatin structure and cancer and to the best of our knowledge are the first to demonstrate a direct role of macroH2 $\mathrm{A}$ in this process.

\section{Acknowledgements}

This study was supported by the Natural Science Foundation of Hunan Provincial (grant no. 13JJ2011) and the Scientific Research Fund of Hunan Provincial Health Department (grant no. hn772705).

\section{References}

1. Portela A and Esteller M: Epigenetic modifications and human disease. Nat Biotechnol 28: 1057-1068, 2010

2. Baylin SB and Jones PA: A decade of exploring the cancer epigenome-biological and translational implications. Nat Rev Cancer 11: 726-734, 2011.

3. Kulaeva OI, Gaykalova DA and Studitsky VM: Transcription through chromatin by RNA polymerase II: histone displacement and exchange. Mutat Res 618: 116-129, 2007.

4. Neely KE and Workman JL: The complexity of chromatin remodeling and its links to cancer. Biochim Biophys Acta 1603: 19-29, 2002.

5. Hake SB, Xiao A and Allis CD: Linking the epigenetic 'language' of covalent histone modifications to cancer. Br J Cancer 90: 761-769, 2004.

6. Strahl BD and Allis CD: The language of covalent histone modifications. Nature 403: 41-45, 2000.

7. Peterson CL and Laniel MA. Histones and histone modifications. Curr Biol 14: R546-R551, 2004.

8. Redon C, Pilch D, Rogakou E, et al: Histone H2a variants H2AX and H2AZ. Curr Opin Genet Dev 12: 162-169, 2002.

9. Pehrson JR and Fried VA: macroH2A, a core histone containing a large nonhistone region. Science 257: 1398-1400, 1992.

10. Kustatscher G, Hothorn M, Pugieux C, et al: Splicing regulates NAD metabolite binding to histone macroH2A. Nat Struct Mol Biol 12: 624-625, 2005.

11. Brooks WH: X chromosome inactivation and autoimmunity. Clin Rev Allergy Immunol 39: 20-29, 2010.

12. Chadwick BP and Willard HF: Histone H2A variants and the inactive $\mathrm{X}$ chromosome: identification of a second macroH2A variant. Hum Mol Genet 10: 1101-1113, 2001.

13. Kapoor A, Goldberg MS, Cumberland LK, et al: The histone variant macroH2A suppresses melanoma progression through regulation of CDK8. Nature 468: 1105-1109, 2010.

14. Rickert P, Seghezzi W, Shanahan F, et al: Cyclin C/CDK8 is a novel CTD kinase associated with RNA polymerase II. Oncogene 12: 2631-2640, 1996.

15. Donner AJ, Szostek S, Hoover JM, et al: CDK8 is a stimulus-specific positive coregulator of p53 target genes. Mol Cell 27: 121-133, 2007.

16. Sánchez I and Dynlacht BD: New insights into cyclins, CDKs, and cell cycle control. Semin Cell Dev Biol: 311-321, 2005. 
17. Satyanarayana A and Kaldis P: Mammalian cell-cycle regulation: several Cdks, numerous cyclins and diverse compensatory mechanisms. Oncogene 28: 2925-2939, 2009.

18. Fisher RP: CDKs and cyclins in transition(s). Curr Opin Genet Dev 7: 32-38, 1997.

19. Kastan MB and Bartek J: Cell-cycle checkpoints and cancer. Nature 432: 316-323, 2004.

20. Andreassi MG: DNA damage, vascular senescence and atherosclerosis. J Mol Med (Berl) 86: 1033-1043, 2008.

21. Morgan DO: Cyclin-dependent kinases: engines, clocks, and microprocessors. Annu Rev Cell Dev Biol 13: 261-291, 1997.

22. Tyson JJ, Csikasz-Nagy A and Novak B: The dynamics of cell cycle regulation. Bioessays 24: 1095-1109, 2002.

23. Sherr CJ and Roberts JM: CDK inhibitors: positive and negative regulators of G1-phase progression. Genes Dev 13: 1501-1512, 1999.

24. Taylor WR and Stark GR: Regulation of the G2/M transition by p53. Oncogene 20: 1803-1815, 2001.

25. Sporn JC, Kustatscher G, Hothorn T, et al: Histone macroH2A isoforms predict the risk of lung cancer recurrence. Oncogene 28: 3423-3428, 2009.

26. Novikov L, Park JW, Chen H, et al: QKI-mediated alternative splicing of the histone variant macroH2 $\mathrm{A} 1$ regulates cancer cell proliferation. Mol Cell Biol 31: 4244-4255, 2011.
27. Haugstetter A, Loddenkemper C, Lenze D, et al: Cellular senescence predicts treatment outcome in metastasised colorectal cancer. Br J Cancer 103: 505-509, 2010.

28. Buschbeck M and Di Croce L: Approaching the molecular and physiological function of macroH2A variants. Epigenetics 5: 118-123, 2010.

29. Zhang R and Adams PD: Heterochromatin and its relationship to cell senescence and cancer therapy. Cell Cycle 6: 784-789, 2007.

30. Collado M, Gil J, Efeyan A, et al: Tumour biology: senescence in premalignant tumours. Nature 436: 642, 2005.

31. Dardenne E, Pierredon S, Driouch K, et al: Splicing switch of an epigenetic regulator by RNA helicases promotes tumor-cell invasiveness. Nat Struct Mol Biol 19: 1139-1146, 2012.

32. Shtutman M, Zhurinsky J, Simcha I, et al: The cyclin D1 gene is a target of the $\beta$-catenin/LEF-1 pathway. Proc Natl Acad Sci USA 96: 5522-5527, 1999.

33. Fu M, Wang C, Li Z, et al: Minireview: Cyclin D1: normal and abnormal functions. Endocrinology 145: 5439-5447, 2004.

34. Hunter T and Pines J: Cyclins and cancer. II: Cyclin D and CDK inhibitors come of age. Cell 79: 573-582, 1994.

35. Gray N, Détivaud L, Doerig C, et al: ATP-site directed inhibitors of cyclin-dependent kinases. Curr Med Chem 6: 859-875, 1999.

36. Lapenna S and Giordano A: Cell cycle kinases as therapeutic targets for cancer. Nat Rev Drug Discov 8: 547-566, 2009. 